\title{
Decay of Potential Vortex and Diffusion of Temperature in a Generalized Oldroyd-B Fluid through a Porous Medium
}

\author{
Xiaoyi Guo \\ School of Science, Linyi University, Linyi, Shandong 276005, China \\ Correspondence should be addressed to Xiaoyi Guo; guoxyi@sina.com \\ Received 10 December 2013; Revised 12 February 2014; Accepted 12 February 2014; Published 30 March 2014 \\ Academic Editor: Ebrahim Momoniat \\ Copyright (C) 2014 Xiaoyi Guo. This is an open access article distributed under the Creative Commons Attribution License, which \\ permits unrestricted use, distribution, and reproduction in any medium, provided the original work is properly cited. \\ Based on a modified Darcy law, the decay of potential vortex and diffusion of temperature in a generalized Oldroyd-B fluid with \\ fractional derivatives through a porous medium is studied. Exact solutions of the velocity and temperature fields are obtained in \\ terms of the generalized Mittag-Leffler function by using the Hankel transform and discrete Laplace transform of the sequential \\ fractional derivatives. One of the solutions is the sum of the Newtonian solutions and the non-Newtonian contributions. As limiting \\ cases of the present solutions, the corresponding solutions of the fractional Maxwell fluid and classical Maxwell fluids are given. \\ The influences of the fractional parameters, material parameters, and the porous space on the decay of the vortex are interpreted \\ by graphical results.
}

\section{Introduction}

In recent years, non-Newtonian fluids that cannot be described by the classical Navier-Stokes equations increasingly play an important role in many industries and technological applications. For the well-known fact that all nonNewtonian fluids are so complicated that they cannot be described by a single constitutive equation, various models of non-Newtonian fluid have been suggested in the literature. Amongst those models, the rate type fluid models, by the pioneering work of Frohlick and Sack [1] and Oldroyd [2], have received much more attention. As one of the rate type models, the Oldroyd-B model [3-6] has succeeded in describing important responses of some polymeric liquids.

Additionally, fractional calculus has been successful in the description of the viscoelastic properties of nonNewtonian fluids. The starting point of the fractional derivative model of a non-Newtonian fluid is usually a classical differential equation, which is modified by replacing the time derivative of an integer order by the fractional calculus operators. These fractional models have been proved to be valuable tools in characterizing the properties of polymeric solutions and melts [7-10].
The studies on vortex flow of viscoelastic fluid and heat flow for viscoelastic fluids are motivated by many practical applications, such as petroleum, chemical, and food industries and bioengineering. The corresponding problem for Newtonian fluids has been investigated by Zierep [11], by means of the similarity transformation, and for second grade fluids it was studied by Fetecau et al. [12]. Later, the results were extended to the generalized second grade fluids [13], fractional Maxwell model [14], Oldroyd-B fluids [15], and generalized Oldroyd-B fluids [16].

Moreover, special attention is given to the flow of viscoelastic fluid through porous media, due to the demands of such diverse areas as biorheology, geophysics, and chemical and petroleum industries [17-19]. In these references, the classical Darcy law was used to describe the effects of the pores on the velocity of a viscoelastic fluid.

In this paper, we consider vortex velocity and temperature fields for a generalized Oldroyd-B fluid with fractional derivatives through porous medium. Based on a modified Darcy law for a viscoelastic fluid, exact solutions are obtained in terms of generalized Mittag-Leffler function by using the Hankel transform and discrete Laplace transform of the sequential fractional derivatives. 


\section{Basic Equations}

We begin by recalling the definition of the constitutive relationship of an incompressible fluid of Oldroyd-B type (also called the Jeffreys model). The extra stress tensor $\mathbf{T}$ is given by the constitutive equation [15]:

$$
\begin{aligned}
\mathbf{T} & =-p \mathbf{I}+\mathbf{S} \\
\mathbf{S}+\lambda \frac{\mathbf{D S}}{\mathbf{D} t} & =\mu\left[1+\lambda_{t} \frac{\mathbf{D}}{\mathbf{D} t}\right] \mathbf{A},
\end{aligned}
$$

where $-p$ I denotes the indeterminate spherical stress due to the constraint of incompressibility, $\mathbf{S}$ is the extra stress tensor, $\mu$ is the viscosity of the fluid, and $\lambda$ and $\lambda_{t}$ are constant relaxation and retardation times, respectively. It is assumed that $\lambda \geq \lambda_{t} \geq 0 . \mathbf{A}=\mathbf{L}+\mathbf{L}^{T}$ is the first Rivlin-Ericksen tensor, $\mathbf{L}=\nabla \mathbf{V}$ is the velocity gradient, $\mathbf{V}$ is the velocity vector, and $\mathbf{D} / \mathbf{D} t$ is defined as follows:

$$
\begin{gathered}
\frac{\mathbf{D S}}{\mathbf{D} t}=\frac{\partial \mathbf{S}}{\partial t}+(\mathbf{V} \cdot \nabla) \mathbf{S}-\mathbf{S}(\nabla \mathbf{V})-(\nabla \mathbf{V})^{T} \mathbf{S} \\
\frac{\mathbf{D A}}{\mathbf{D} t}=\frac{\partial \mathbf{A}}{\partial t}+(\mathbf{V} \cdot \nabla) \mathbf{A}-\mathbf{A}(\nabla \mathbf{V})-(\nabla \mathbf{V})^{T} \mathbf{A}
\end{gathered}
$$

Due to the symmetry of the considered circular motion for an Oldroyd-B fluid, the velocity field, in a system of cylindrical coordinates $(r, \theta, z)$, can be assumed as $v_{r}=$ $0, v_{\theta}=\omega(r, t)$, and $v_{z}=0$. This flow field automatically satisfies the constraint of incompressibility. We suppose the initial distribution of the velocity is that of a potential of circulation $\Gamma_{0}$ and the flow is symmetrical with respect to the axis through a porous medium. Having in mind the initial condition $\mathbf{S}(r, 0)=0$, we can obtain the constitutive relationship:

$$
\left(1+\lambda \partial_{t}\right) S_{r \theta}=\mu\left(1+\lambda_{t} \partial_{t}\right) r \frac{\partial}{\partial r}\left(\frac{\omega}{r}\right)
$$

for the Oldroyd-B fluid performing this flow.

A direct approach to create fractional rheological constitutive equations is to replace the regular time derivatives of ordinary equation by fractional time derivatives of noninteger order. Therefore, according to the constitutive relationship of the classical Oldroyd-B fluid, the constitutive relationship of the generalized Oldroyd- $\mathrm{B}$ is given by

$$
\left(1+\lambda D_{t}^{\alpha}\right) S_{r \theta}=\mu\left(1+\lambda_{t} D_{t}^{\beta}\right) r \frac{\partial}{\partial r}\left(\frac{\omega}{r}\right),
$$

where $D_{t}^{\alpha}$ and $D_{t}^{\beta}$ are fractional derivatives of order $\alpha$ and $\beta$ with respect to $t$, respectively, and are defined as [20]

$$
D_{t}^{p} f(t)=\frac{1}{\Gamma(1-p)} \int_{0}^{t}(t-\tau)^{-p} f^{\prime}(\tau) d \tau, \quad 0 \leq p \leq 1 .
$$

The new material constants $\lambda$ and $\lambda_{t}$ in (4) have the dimensions of $t^{\alpha}$ and $t^{\beta}$, respectively. Some of the papers use $\lambda^{\alpha}$ and $\lambda^{\beta}$ instead of $\lambda$ and $\lambda_{t}$. However, for the sake of simplicity, we keep the same notations as in the ordinary case. This model includes the ordinary Oldroyd-B fluid as a special case for $\alpha=\beta=1$ and includes the fractional Maxwell and classical Maxwell fluid for $\lambda_{t} \rightarrow 0, \beta=1$ and for $\lambda_{t} \rightarrow 0, \alpha=\beta=1$, respectively.

It is well known that in the flow of viscous Newtonian fluid at a low speed through a porous medium the pressure drop caused by the frictional drag is directly proportional to velocity, which is the Darcy law. Recently many modified Darcy's laws have been presented for viscous Newtonian fluid flows in porous media. However, for viscoelastic fluid in porous media, only a few mathematical macroscopic filtration models were proposed. By analogy with OldroydB fluid's constitutive relationship, the following phenomenological model, which relates pressure drop and velocity for a viscoelastic fluid in an unbounded porous medium, has been introduced [17]:

$$
\left(1+\lambda \frac{\partial}{\partial t}\right) \nabla p=-\frac{\mu \phi}{K}\left(1+\lambda_{t} \frac{\partial}{\partial t}\right) \mathbf{V}
$$

where $K$ is the permeability and $\phi$ is the porosity of the porous medium. Equation (6) can be simplified to Darcy's law when $\lambda=\lambda_{t}=0$. In the same way, by analogy with generalized Oldroyd-B fluid's constitutive relationship equation (4), we introduce a modified phenomenological Darcy law with fractional derivatives as follows:

$$
\left(1+\lambda D_{t}^{\alpha}\right) \nabla p=-\frac{\mu \phi}{K}\left(1+\lambda_{t} D_{t}^{\beta}\right) \mathbf{V}
$$

In consideration of the balance of forces acting on a volume element of fluid, the local volume average balance of linear momentum can be given by

$$
\rho \frac{d \mathbf{V}}{d t}=-\nabla p+\operatorname{div} \mathbf{S}+\mathbf{r}
$$

where $d / d t$ is the material time derivative, $\rho$ denotes the density, and $\mathbf{r}$ is Darcy's resistance for a generalized Oldroyd$B$ fluid in the porous medium and can be inferred from (7) to satisfy the following equation [17]:

$$
\left(1+\lambda D_{t}^{\alpha}\right) \mathbf{r}=-\frac{\mu \phi}{K}\left(1+\lambda_{t} D_{t}^{\beta}\right) \mathbf{V}
$$

Then we obtain the momentum equation through a porous medium in the form:

$$
\begin{aligned}
\rho\left(1+\lambda D_{t}^{\alpha}\right) \frac{\partial \omega(r, t)}{\partial t}= & \left(1+\lambda D_{t}^{\alpha}\right) \frac{1}{r^{2}} \frac{\partial}{\partial r}\left(r^{2} S_{r \theta}\right) \\
& -\frac{\mu \phi}{K}\left(1+\lambda_{t} D_{t}^{\beta}\right) \omega(r, t),
\end{aligned}
$$

where the pressure gradient in the $\theta$ direction is ignored.

Substituting (4) into (10), we obtain

$$
\begin{aligned}
\left(1+\lambda D_{t}^{\alpha}\right) \frac{\partial \omega}{\partial t}= & \nu\left(1+\lambda_{t} D_{t}^{\beta}\right)\left(\frac{\partial^{2}}{\partial r^{2}}+\frac{1}{r} \frac{\partial}{\partial r}-\frac{1}{r^{2}}\right) \omega \\
& -\frac{\nu \phi}{K}\left(1+\lambda_{t} D_{t}^{\beta}\right) \omega,
\end{aligned}
$$


in which $\nu=\mu / \rho$ is the kinematic viscosity. The initial and natural conditions are

$$
\begin{gathered}
\omega(r, 0)=\frac{\Gamma_{0}}{2 \pi r}, \quad \frac{\partial \omega}{\partial t}(r, 0)=0, \\
\omega(r, t), \frac{\partial \omega(r, t)}{\partial r} \longrightarrow 0, \quad \text { as } r \longrightarrow \infty, t>0 .
\end{gathered}
$$

Further, we consider that there exists temperature field during the vortex flow in a generalized Oldroyd-B fluid. Its initial distribution and natural conditions are similarly assumed to be in the same form of previous studies $[13,18]$ with

$$
\begin{gathered}
\theta(r, 0)=\frac{\theta_{0}}{2 \pi r}, \\
\theta(r, t), \frac{\partial \theta(r, t)}{\partial r} \longrightarrow 0, \quad \text { as } r \longrightarrow \infty, t>0 .
\end{gathered}
$$

The energy equation, when the Fourier law of heat conduction is applied, may be written in the form $[13,18]$ :

$$
\begin{aligned}
\frac{\partial \theta(r, t)}{\partial t}= & \frac{k}{\rho c}\left(\frac{\partial^{2}}{\partial r^{2}}+\frac{\partial}{r \partial r}\right) \theta(r, t) \\
& +\frac{\nu}{c}\left[\frac{\partial \omega(r, t)}{\partial r}-\frac{\omega(r, t)}{r}\right]^{2}+\frac{h(r, t)}{c},
\end{aligned}
$$

where $h(r, t)$ is the radiant heating which is neglected in this paper, $c$ is the specific heat, and $k$ is the conductivity (be assumed to be constant).

\section{Velocity Field}

Let us introduce dimensionless variables $\omega^{*}=\omega \nu / \Gamma_{0}^{2}, r^{*}=$ $r \Gamma_{0} / \nu$, and $t^{*}=t \Gamma_{0}^{2} / \nu$ and dimensionless parameters $\lambda^{*}=$ $\lambda\left(\Gamma_{0}^{2} / \nu\right)^{\alpha}$ and $\lambda_{t}^{*}=\lambda_{t}\left(\Gamma_{0}^{2} / \nu\right)^{\beta}$. Then (11) and (12) can be changed into dimensionless equations as follows (for brevity the dimensionless mark " $*$ " is omitted here):

$$
\begin{aligned}
& \left(1+\lambda D_{t}^{\alpha}\right) \frac{\partial \omega}{\partial t}=\left(1+\lambda_{t} D_{t}^{\beta}\right)\left(\frac{\partial^{2}}{\partial r^{2}}+\frac{1}{r} \frac{\partial}{\partial r}-\frac{1}{r^{2}}\right) \omega \\
& -\eta^{2}\left(1+\lambda_{t} D_{t}^{\beta}\right) \omega \\
& \omega(r, 0)=\frac{1}{2 \pi r}, \quad \frac{\partial \omega}{\partial t}(r, 0)=0, \\
& \omega(r, t), \frac{\partial \omega(r, t)}{\partial r} \longrightarrow 0, \quad \text { as } r \longrightarrow \infty, t>0,
\end{aligned}
$$

where $\eta=1 / \operatorname{Re}\left(\operatorname{Re}=\Gamma_{0} \sqrt{K / \phi} / \nu\right.$ is the Reynolds number in porous media).

In order to get the exact solution of above equations, we introduce the Hankel transform of order $n$ and its inverse transform as follows [21]:

$$
\begin{aligned}
& \bar{f}(\xi)=H_{n}[f(r) ; \xi]=\int_{0}^{\infty} r J_{n}(\xi r) f(r) d r, \\
& f(r)=H_{n}^{-1}[\bar{f}(\xi) ; r]=\int_{0}^{\infty} \xi J_{n}(\xi r) \bar{f}(\xi) d \xi,
\end{aligned}
$$

where $J_{n}(\xi r)$ is the first kind of the Bessel function of order $n$. Applying the Hankel transform of order one $(n=1)$ to (16) and (17) and having in mind condition (18), we can obtain

$$
\begin{gathered}
\left(1+\lambda D_{t}^{\alpha}\right) \frac{\partial \bar{\omega}}{\partial t}+\left(\xi^{2}+\eta^{2}\right)\left(1+\lambda_{t} D_{t}^{\beta}\right) \bar{\omega}=0, \\
\bar{\omega}(\xi, 0)=\frac{1}{2 \pi \xi}, \quad \frac{\partial \bar{\omega}}{\partial t}(\xi, 0)=0 .
\end{gathered}
$$

The Laplace transform is defined as follows:

$$
\widetilde{\bar{\omega}}(\xi, s)=L[\bar{\omega}(\xi, t) ; s]=\int_{0}^{\infty} e^{-s t} \bar{\omega}(\xi, t) d t .
$$

Then, applying the Laplace transform to (17) and considering the formula [19]:

$$
\begin{array}{r}
L\left[D_{t}^{q} f(t) ; s\right]=s^{q} \tilde{f}(s)-\sum_{k=0}^{n-1} s^{q-k-1} f^{(k)}(0), \\
n-1<q \leq n,
\end{array}
$$

we get

$$
\widetilde{\bar{\omega}}(\xi, s)=\frac{1}{2 \pi \xi} \frac{\lambda s^{\alpha}+1+\left(\xi^{2}+\eta^{2}\right) \lambda_{t} s^{\beta-1}}{\lambda s^{\alpha+1}+s+\left(\xi^{2}+\eta^{2}\right) \lambda_{t} s^{\beta}+\xi^{2}+\eta^{2}} .
$$

In order to avoid the burdensome calculations of residues and contour integrals, we apply the discrete inverse Laplace transform method to give $\bar{\omega}(\xi, t)$. Firstly, we rewrite (23) as a series form

$$
\begin{aligned}
\tilde{\bar{\omega}}(\xi, s)=\frac{1}{2 \pi \xi}[ & \frac{1}{s}-\sum_{k=0}^{\infty}(-1)^{k}\left(\frac{\xi^{2}+\eta^{2}}{\lambda}\right)^{k+1} \\
& \left.\times \sum_{m=0}^{k}\left(\begin{array}{c}
k \\
m
\end{array}\right) \lambda_{t}^{m} \frac{s^{\beta m-k-2}}{\left(s^{\alpha}+\lambda^{-1}\right)^{k+1}}\right] .
\end{aligned}
$$

Then, applying the inverse Laplace transform term by term to (24) and considering the following property of the generalized Mittag-Leffler function [20]:

$$
L^{-1}\left[\frac{n ! s^{\lambda-\mu}}{\left(s^{\lambda} \mp c\right)^{n+1}}\right]=t^{\lambda n+\mu-1} E_{\lambda, \mu}^{(n)}\left( \pm c t^{\lambda}\right), \quad \operatorname{Re}(s)>|c|^{1 / \lambda}
$$

we have

$$
\begin{aligned}
\bar{\omega}(\xi, t)=\frac{1}{2 \pi \xi}[1 & -\sum_{k=0}^{\infty} \frac{(-1)^{k}\left(\xi^{2}+\eta^{2}\right)^{k+1}}{k ! \lambda^{k+1}} \\
& \times \sum_{m=0}^{k}\left(\begin{array}{c}
k \\
m
\end{array}\right) \lambda_{t}^{m} t^{\alpha k+\alpha-\beta m+k+1} \\
& \left.\times E_{\alpha, \alpha-\beta m+k+2}^{(k)}\left(-\lambda^{-1} t^{\alpha}\right)\right] .
\end{aligned}
$$


Finally, applying the inverse Hankel transform to (26), we obtain the exact solution to the velocity field

$$
\begin{aligned}
& \omega(r, t) \\
& =\frac{1}{2 \pi} \int_{0}^{\infty} J_{1}(\xi r) d \xi \\
& \quad-\frac{1}{2 \pi} \int_{0}^{\infty} \sum_{k=0}^{\infty} \sum_{m=0}^{k} \frac{(-1)^{k}\left(\xi^{2}+\eta^{2}\right)^{k+1} \lambda_{t}^{m} t^{\alpha k+\alpha-\beta m+k+1}}{m !(k-m) ! \lambda^{k+1}} \\
& \times E_{\alpha, \alpha-\beta m+k+2}^{(k)}\left(-\lambda^{-1} t^{\alpha}\right) J_{1}(\xi r) d \xi .
\end{aligned}
$$

In addition, we can also rewrite (23) as another form of series as follows:

$$
\begin{aligned}
\widetilde{\bar{\omega}}(\xi, s)= & \frac{1}{2 \pi \xi} \frac{1}{s+\xi^{2}+\eta^{2}} \\
& +\frac{\xi^{2}+\eta^{2}}{2 \pi \xi} \frac{1}{s+\xi^{2}+\eta^{2}} \\
& \times \sum_{k=0}^{\infty} \sum_{m=0}^{k} \frac{(-1)^{k}\left(\xi^{2}+\eta^{2}\right)^{k} \lambda_{t}^{m} k !}{\lambda^{k+1} m !(k-m) !} \\
& \times\left[\lambda \frac{s^{\alpha+\beta m-(k+1)}}{\left(s^{\alpha}+\lambda^{-1}\right)^{k+1}}\right. \\
\quad+\lambda_{t}\left(\xi^{2}+\eta^{2}\right) & \left.\frac{s^{\beta+\beta m-(k+2)}}{\left(s^{\alpha}+\lambda^{-1}\right)^{k+1}}\right] .
\end{aligned}
$$

Applying the discrete inverse Laplace transform to the above equation and considering (25), we get

$$
\bar{\omega}(\xi, t)=\frac{1}{2 \pi \xi} e^{-t\left(\xi^{2}+\eta^{2}\right)}+\frac{\xi^{2}+\eta^{2}}{2 \pi \xi} \int_{0}^{t} e^{-(t-s)\left(\xi^{2}+\eta^{2}\right)} F(\xi, s) d s,
$$

where

$$
\begin{aligned}
F(\xi, t)=\sum_{k=0}^{\infty} \sum_{m=0}^{k} \frac{(-1)^{k}\left(\xi^{2}+\eta^{2}\right)^{k} \lambda_{t}^{m} t^{\alpha k+k-\beta m}}{\lambda^{k} m !(k-m) !} \\
\quad \times E_{\alpha, k+1-\beta m}^{(k)}\left(-\lambda^{-1} t^{\alpha}\right) \\
+\sum_{k=0}^{\infty} \sum_{m=0}^{k} \frac{(-1)^{k}\left(\xi^{2}+\eta^{2}\right)^{k+1} \lambda_{t}^{m+1} t^{\alpha k+k+\alpha+1-\beta-\beta m}}{\lambda^{k+1} m !(k-m) !} \\
\quad \times E_{\alpha, \alpha+k+2-\beta-\beta m}^{(k)}\left(-\lambda^{-1} t^{\alpha}\right) .
\end{aligned}
$$

Then, applying the inverse Hankel transform to (29), we obtain the equivalent solution of vortex velocity as follows:

$$
\begin{aligned}
& \omega(r, t) \\
& =\omega_{\mathrm{NP}}(r, t) \\
& \quad+\frac{1}{2 \pi} \int_{0}^{\infty} \int_{0}^{t}\left(\xi^{2}+\eta^{2}\right) e^{-(t-s)\left(\xi^{2}+\eta^{2}\right)} F(\xi, s) J_{1}(\xi r) d s d \xi
\end{aligned}
$$

where (cf. [19, equation (41)])

$$
\omega_{\mathrm{NP}}(r, t)=\frac{1}{2 \pi} \int_{0}^{\infty} e^{-t\left(\xi^{2}+\eta^{2}\right)} J_{1}(\xi r) d \xi
$$

is the velocity field corresponding to a Newtonian fluid performing the same motion through a porous medium.

\section{Temperature Field}

Equations (13) (15) can be nondimensionalized by introducing dimensionless variables $\theta^{*}=\theta v / \theta_{0} \Gamma_{0}, \omega^{*}=\omega \nu / \Gamma_{0}^{2}, r^{*}=$ $r \Gamma_{0} / \nu$, and $t^{*}=t \Gamma_{0}^{2} / \nu$. The dimensionless equation with the initial and natural boundary conditions can be given as follows (for brevity, the dimensionless mark “*” is omitted here):

$$
\begin{gathered}
\frac{\partial \theta(r, t)}{\partial t}=\beta_{1}\left(\frac{\partial^{2}}{\partial r^{2}}+\frac{\partial}{r \partial r}\right) \theta(r, t)+\eta_{1} f(r, t), \\
\theta(r, 0)=\frac{1}{2 \pi r}, \\
\theta(r, t), \frac{\partial \theta(r, t)}{\partial r} \longrightarrow 0, \quad \text { as } r \longrightarrow \infty, t>0,
\end{gathered}
$$

where $\beta_{1}=k / \mu c, \eta_{1}=\Gamma_{0}^{3} / c \nu \theta_{0}$, and

$$
f(r, t)=\left[\frac{\partial \omega(r, t)}{\partial r}-\frac{\omega(r, t)}{r}\right]^{2}
$$

Making the Hankel transform of zero order $(n=0)$ with respect to $r$ in (33) and (34), and having in mind the condition (35), we obtain

$$
\begin{gathered}
\frac{\partial \bar{\theta}(\xi, t)}{\partial t}+\beta_{1} \xi^{2} \bar{\theta}(\xi, t)=\eta_{1} \bar{f}(\xi, t), \\
\theta(r, 0)=\frac{1}{2 \pi \xi},
\end{gathered}
$$


in which $\bar{f}(\xi, t)$ is given by

$$
\begin{aligned}
& \bar{f}(\xi, t) \\
& =\frac{1}{4 \pi^{2}} \\
& \times \int_{0}^{\infty} r J_{0}(\xi r) \\
& \times\left\{\int_{0}^{\infty} x J_{2}(x r)\right. \\
& \times\left[1-\sum_{k=0}^{\infty} \sum_{m=0}^{k}\left((-1)^{k}\left(\xi^{2}+\eta^{2}\right)^{k+1}\right.\right. \\
& \left.\times \lambda_{t}^{m} t^{\alpha k+\alpha-\beta m+k+1}\right) \\
& \left.\times\left(m !(k-m) ! \lambda^{k+1}\right)^{-1}\right) \\
& \times E_{\alpha, \alpha-\beta m+k+2}^{(k)} \\
& \left.\left.\times\left(-\lambda^{-1} t^{\alpha}\right)\right] d x\right\}^{2} d r .
\end{aligned}
$$

To obtain the equation above, we substituted (27) into (36) and used the following property of the Bessel function:

$$
x J_{1}^{\prime}(x)=J_{1}(x)-x J_{2}(x) .
$$

Solving (37) with (38), we have

$$
\bar{\theta}(\xi, t)=e^{-\beta_{1} \xi^{2} t}\left[\frac{1}{2 \pi \xi}+\eta_{1} \int_{0}^{t} \bar{f}(\xi, \tau) e^{\beta_{1} \xi^{2} \tau} d \tau\right] .
$$

Then, applying the inverse Hankel transform of zero order to (41), we get the exact solution to the temperature field

$$
\begin{aligned}
& \theta(r, t) \\
& \quad=\int_{0}^{\infty} J_{0}(\xi r) e^{-\beta_{1} \xi^{2} t}\left[\frac{1}{2 \pi}+\eta_{1} \xi \int_{0}^{t} \bar{f}(\xi, \tau) e^{\beta_{1} \xi^{2} \tau} d \tau\right] d \xi,
\end{aligned}
$$

in which $\bar{f}(\xi, t)$ is given by (39).

\section{Special Cases and Numerical Results}

When $\eta=0$, (27) and (31) can be simplified to the dimensionless solutions of vortex velocity field for generalized Oldroyd-B fluids in nonporous cases. Equivalent expression for velocity in terms of $G$ function is obtained by Fetecau et al. [16]. And when $\alpha=1, \beta=1$, the above results can be simplified to the solution for ordinary Oldroyd-B fluid through porous medium.
Making $\lambda_{t} \rightarrow 0, \beta=1$ to (27) and (42), we have vortex velocity of the fractional Maxwell fluid through porous medium as follows:

$$
\begin{aligned}
& \omega(r, t) \\
& =\frac{1}{2 \pi} \int_{0}^{\infty} J_{1}(\xi r) d \xi \\
& \quad-\frac{1}{2 \pi} \int_{0}^{\infty} \sum_{k=0}^{\infty} \frac{(-1)^{k}\left(\xi^{2}+\eta^{2}\right)^{k+1} t^{\alpha k+\alpha+k+1}}{k ! \lambda^{k+1}} \\
& =\frac{1}{2 \pi} \int_{0}^{\infty} J_{1}^{(k)}(\xi r) d \xi \\
& \quad-\frac{1}{2 \pi} \int_{0}^{\infty} \sum_{k=0}^{\infty} \sum_{n=0}^{\infty} \frac{(-1)^{k+n}\left(\xi^{2}+\eta^{2}\right)^{k+1}(k+n) ! t^{\alpha k+\alpha n+\alpha+k+1}}{k ! \lambda^{k+n+1} \Gamma(\alpha k+\alpha n+\alpha+k+2)} \\
& \times J_{1}(\xi r) d \xi
\end{aligned}
$$

and the temperature field of the fluid as

$$
\begin{aligned}
& \theta(r, t) \\
& \quad=\int_{0}^{\infty} J_{0}(\xi r) e^{-\beta_{1} \xi^{2} t}\left[\frac{1}{2 \pi}+\eta_{1} \xi \int_{0}^{t} \bar{f}(\xi, \tau) e^{\beta_{1} \xi^{2} \tau} d \tau\right] d \xi,
\end{aligned}
$$

in which $\bar{f}(\xi, t)$ is given by

$\bar{f}(\xi, t)$

$$
\begin{aligned}
=\frac{1}{4 \pi^{2}} \int_{0}^{\infty} r J_{0}(\xi r) & \\
\times & \left\{\int_{0}^{\infty} x J_{2}(x r)\right. \\
\times & \\
& \\
& \times\left(k+\sum_{k=0}^{\infty} \sum_{n=0}^{\infty}\left((-1)^{k+n}\left(\xi^{2}+\eta^{2}\right)^{k+1}\right.\right. \\
& \left.\times t^{\alpha k+\alpha n+\alpha+k+1}\right) \\
& \left.\left.\times\left(k ! \lambda^{k+n+1} \Gamma\right)\right] d x\right\}^{2} d r .
\end{aligned}
$$


Also when making $\lambda_{t} \rightarrow 0, \beta=1$ to (31), we get the solutions

$$
\begin{aligned}
\omega(r, t)=\omega_{\mathrm{NP}}(r, t) & \begin{aligned}
+\frac{1}{2 \pi} \int_{0}^{\infty} \int_{0}^{t} \sum_{k=0}^{\infty} \sum_{n=0}^{\infty}( & \left.-\frac{1}{\lambda}\right)^{k+n} \\
& \times \frac{\left(\xi^{2}+\eta^{2}\right)^{k+1} s^{\alpha k+\alpha n+k}}{n ! k ! \Gamma(\alpha k+\alpha n+k+1)} \\
& \times e^{-(t-s)\left(\xi^{2}+\eta^{2}\right)} J_{1}(\xi r) d s d \xi,
\end{aligned}
\end{aligned}
$$

for the fractional Maxwell fluid through porous medium. When $\eta \rightarrow 0$, (46) is the same result as that obtained by Fetecau et al. [16].

Further making $\alpha=1$ to (43), (44), and (45), we, respectively, have the velocity field

$$
\begin{aligned}
& \omega(r, t) \\
& =\frac{1}{2 \pi} \int_{0}^{\infty} J_{1}(\xi r) d \xi \\
& -\frac{1}{2 \pi} \int_{0}^{\infty} \sum_{k=0}^{\infty} \sum_{n=0}^{\infty} \frac{(-1)^{k+n}\left(\xi^{2}+\eta^{2}\right)^{k+1}(k+n) ! t^{2 k+n+2}}{k ! \lambda^{k+n+1} \Gamma(2 k+n+3)} \\
& \times J_{1}(\xi r) d \xi
\end{aligned}
$$

and the temperature field

$$
\begin{aligned}
& \theta(r, t) \\
& \quad=\int_{0}^{\infty} J_{0}(\xi r) e^{-\beta_{1} \xi^{2} t}\left[\frac{1}{2 \pi}+\eta_{1} \xi \int_{0}^{t} \bar{f}(\xi, \tau) e^{\beta_{1} \xi^{2} \tau} d \tau\right] d \xi
\end{aligned}
$$

where

$$
\begin{aligned}
& \bar{f}(\xi, t) \\
& =\frac{1}{4 \pi^{2}} \int_{0}^{\infty} r J_{0}(\xi r) \\
& \times\left\{\int_{0}^{\infty} x J_{2}(x r)\right. \\
& \times\left[1-\sum_{k=0}^{\infty} \sum_{n=0}^{\infty}\left((-1)^{k+n}\left(\xi^{2}+\eta^{2}\right)^{k+1}\right.\right. \\
& \left.\times(k+n) ! t^{2 k+n+2}\right) \\
& \left.\left.\left.\times\left(k ! \lambda^{k+n+1} \Gamma\right)^{-1}\right)\right] d x\right\}^{2} d r \\
& \times(2 k+n
\end{aligned}
$$

for the classical Maxwell fluid through porous medium.
When $\lambda \rightarrow 0$, (23) reduces to

$$
\widetilde{\bar{\omega}}(\xi, s)=\frac{1}{2 \pi \xi} \frac{1+\left(\xi^{2}+\eta^{2}\right) \lambda_{t} s^{\beta-1}}{s+\left(\xi^{2}+\eta^{2}\right) \lambda_{t} s^{\beta}+\xi^{2}+\eta^{2}} .
$$

Separately applying the inverse Laplace and inverse Hankel transform to $s$ and $\xi$ in the above equation, we have the solution of vortex velocity field

$$
\begin{aligned}
& \omega(r, t)=\frac{1}{2 \pi} \int_{0}^{\infty} \sum_{k=0}^{\infty} \frac{(-t)^{k}\left(\xi^{2}+\eta^{2}\right)^{k}}{k !} \\
& \times E_{1-\beta, \beta k+1}^{(k)}\left(-\lambda_{t}\left(\xi^{2}+\eta^{2}\right)\right. \\
&\left.\times t^{1-\beta}\right) J_{1}(\xi r) d \xi \\
& \times \frac{1}{2 \pi} \int_{0}^{\infty} \sum_{k=0}^{\infty} \frac{(-t)^{k}\left(\xi^{2}+\eta^{2}\right)^{k+1} \lambda_{t} t^{1-\beta}}{k !} \\
& \times E_{1-\beta, \beta k-\beta+2}^{(k)}\left(-\lambda_{t}\left(\xi^{2}+\eta^{2}\right)\right. \\
&\left.\times t^{1-\beta}\right) J_{1}(\xi r) d \xi,
\end{aligned}
$$

which is the same as the result obtained by Shen et al. [13], when $\eta \rightarrow 0$, and temperature field is

$$
\begin{aligned}
& \theta(r, t) \\
& \quad=\int_{0}^{\infty} J_{0}(\xi r) e^{-\beta_{1} \xi^{2} t}\left[\frac{1}{2 \pi}+\eta_{1} \xi \int_{0}^{t} \bar{f}(\xi, \tau) e^{\beta_{1} \xi^{2} \tau} d \tau\right] d \xi,
\end{aligned}
$$

where

$\bar{f}(\xi, t)$

$$
\begin{aligned}
\frac{1}{4 \pi^{2}} \int_{0}^{\infty} r J_{0}(\xi r) & \\
\times & \left\{\int_{0}^{\infty} x J_{2}(x r)\right. \\
& \times\left[\sum_{k=0}^{\infty} \frac{(-t)^{k}\left(\xi^{2}+\eta^{2}\right)^{k}}{k !}\right.
\end{aligned}
$$

$$
\times E_{1-\beta, \beta k+1}^{(k)}\left(-\lambda_{t}\left(\xi^{2}+\eta^{2}\right) t^{1-\beta}\right)
$$




$$
\begin{aligned}
&+\sum_{k=0}^{\infty} \frac{(-t)^{k}\left(\xi^{2}+\eta^{2}\right)^{k+1} \lambda_{t} t^{1-\beta}}{k !} \\
& \times E_{1-\beta, \beta k-\beta+2}^{(k)}(-\lambda_{t}\left(\xi^{2}+\eta^{2}\right) \\
&\left.\left.\left.\times t^{1-\beta}\right)\right] d x\right\}^{2} d r
\end{aligned}
$$

for the generalized second grade fluid through a porous medium.

Making $\lambda_{t} \rightarrow 0, \beta=1$ to (51), (52), and (53), we find the velocity

$$
\begin{aligned}
\omega(r, t) & =\frac{1}{2 \pi} \int_{0}^{\infty} J_{1}(\xi r) \sum_{k=0}^{\infty} \frac{(-t)^{k}\left(\xi^{2}+\eta^{2}\right)^{k} t^{k}}{k !} d \xi \\
& =\frac{1}{2 \pi} \int_{0}^{\infty} J_{1}(\xi r) e^{-\left(\xi^{2}+\eta^{2}\right) t} d \xi
\end{aligned}
$$

and the temperature field

$$
\begin{aligned}
& \theta(r, t)=\int_{0}^{\infty} J_{0}(\xi r) e^{-\beta_{1} \xi^{2} t} \\
& \times\left[\frac{1}{2 \pi}+\eta_{1} \xi\right. \\
& \quad \times \int_{0}^{t} \frac{1}{4 \pi^{2}} \int_{0}^{\infty} r J_{0}(\xi r) \\
& \quad \times\left\{\int_{0}^{\infty} x J_{2}(x r)\right. \\
&\left.\left.\quad \times e^{-\left(\xi^{2}+\eta^{2}\right) t} d x\right\}^{2} d r e^{\beta_{1} \xi^{2} \tau} d \tau\right] d \xi,
\end{aligned}
$$

which correspond to the results for a Newtonian fluid performing the same motion through a porous medium.

In addition, the influences of fractional orders and material parameters on the decay of vortex velocity are also analyzed in graphical form for generalized Oldroyd-B fluids through porous medium. In Figure 1, the velocity field corresponding to (27) is drawn against $r$ for different values of $t$. The influences of the fractional parameters QUOTE $\alpha \alpha$ and $\beta$ on the decay of the vortex are showed in Figures 2 and 3 . The influences of the material parameters $\lambda$ and $\lambda_{t}$ on the decay of the vortex are showed in Figures 4 and 5, respectively. In order to compare the vortex velocities for fractional Oldroyd-B, fractional Maxwell, and Newtonian fluids performing the same motion in porous and nonporous media, we draw Figures 6 and 7, respectively.

\section{Conclusions}

In this paper, by analogy to constitutive relationship of the generalized Oldroyd-B fluid, we introduce a modified phenomenological Darcy law with fractional derivative. Based on

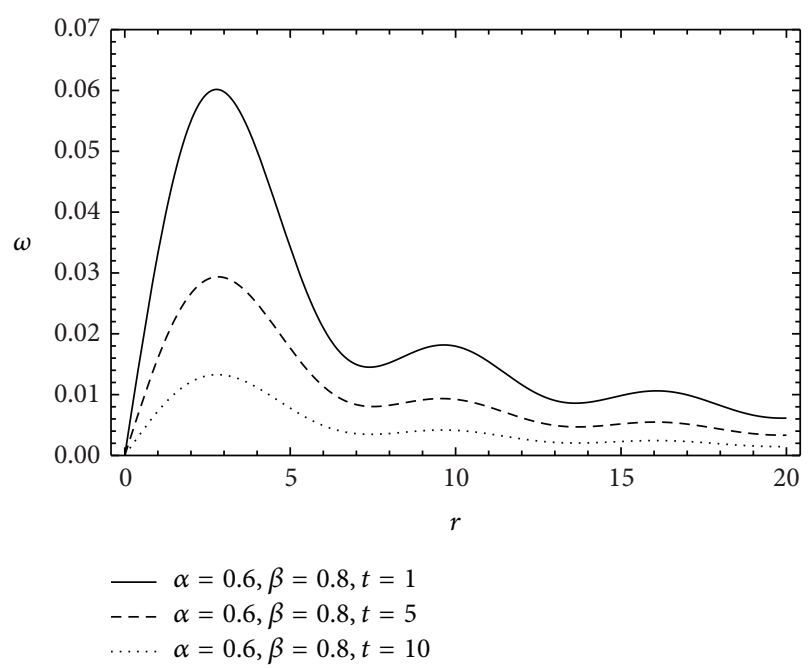

FIGURE 1: Profiles of velocity $\omega(r, t)$ versus $r$ for different values of $t$, for $\lambda=6, \lambda_{t}=3$.

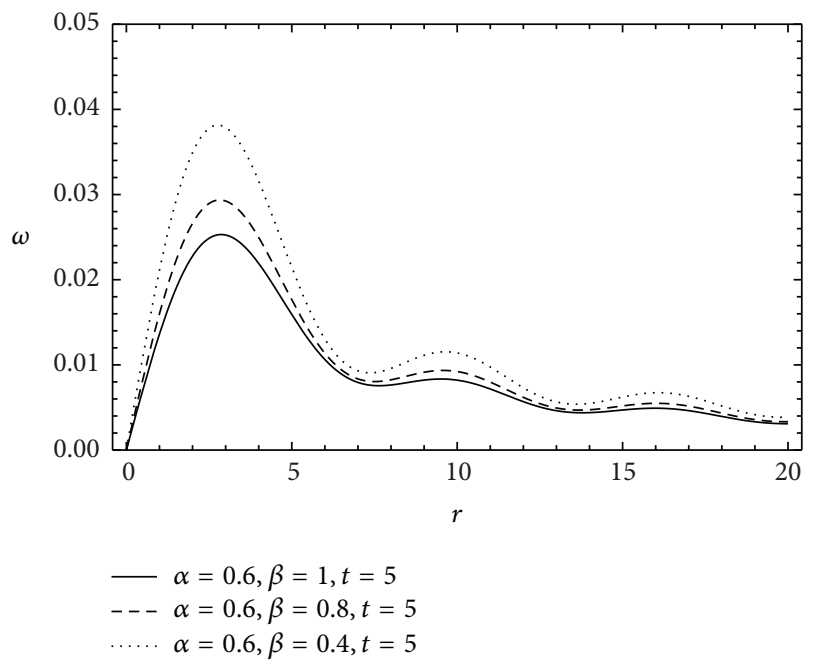

Figure 2: Profiles of velocity $\omega(r, t)$ versus $r$ for different values of $\beta$, for $\lambda=6, \lambda_{t}=3$.

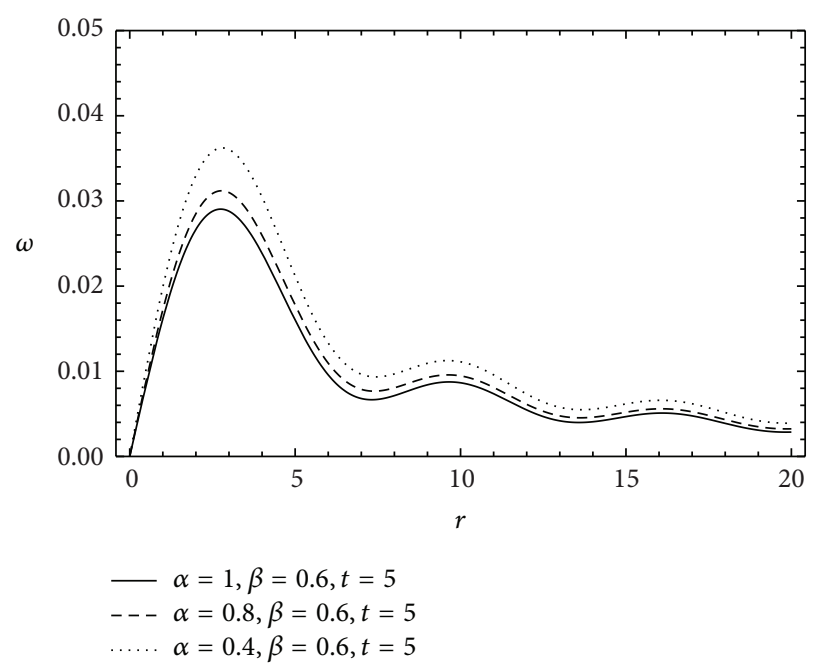

FIgURE 3: Profiles of velocity $\omega(r, t)$ versus $r$ for different values of $\alpha$, for $\lambda=6, \lambda_{t}=3$. 


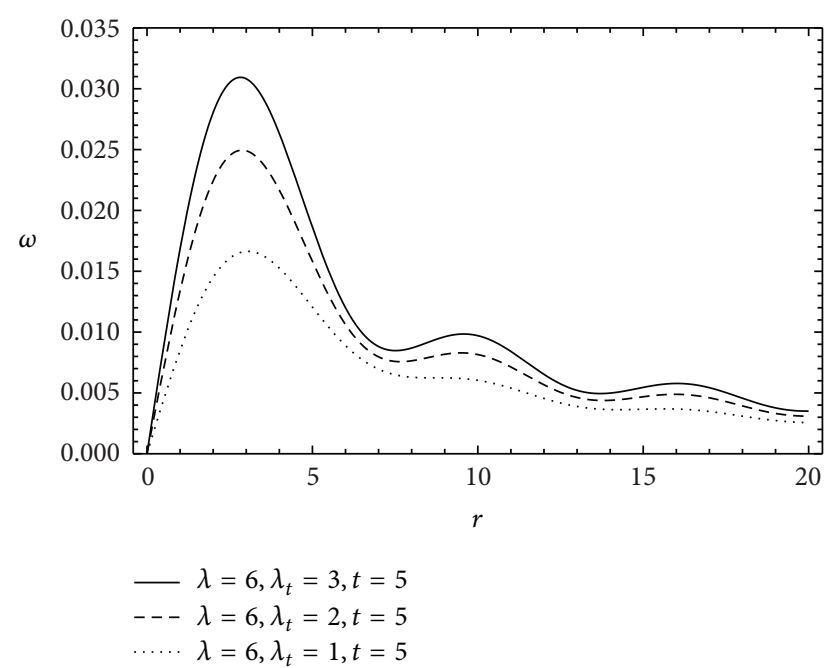

FIGURE 4: Profiles of velocity $\omega(r, t)$ versus $r$ for different values of $\lambda_{t}$, for $\alpha=0.5, \beta=0.8$.

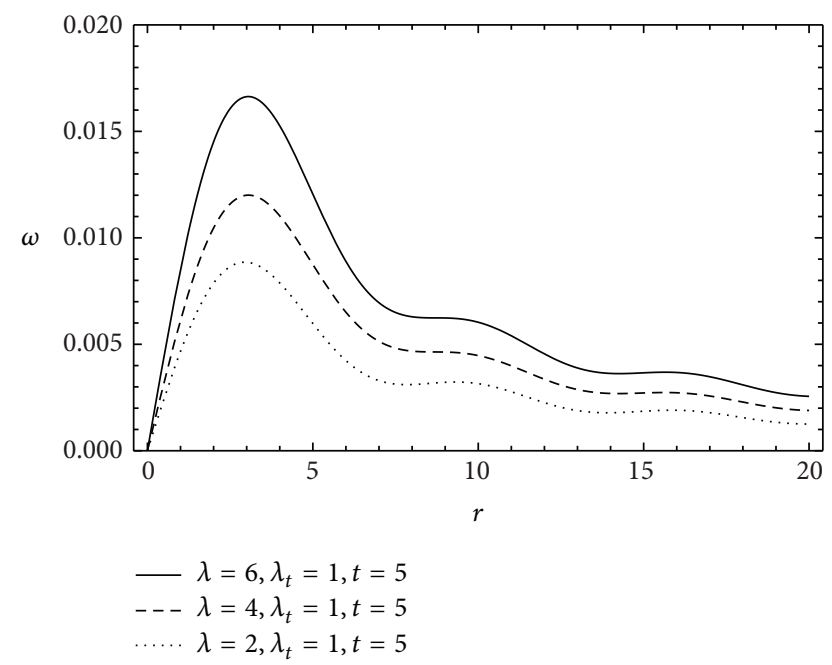

FIGURE 5: Profiles of velocity $\omega(r, t)$ versus $r$ for different values of $\lambda$, for $\alpha=0.5, \beta=0.8$.

this model, the decay of a potential vortex and diffusion of temperature in a generalized Oldroyd-B fluid with fractional derivatives through a porous medium is studied. Using the Laplace transform and Hankel transform, we provide the exact solutions of the velocity and temperature fields. Particularly, we give the solution of vortex velocity of two equivalent forms, one of which is the sum of the Newtonian solutions and the non-Newtonian contributions. And the results for decay of a potential vortex in Oldroyd-B, generalized Maxwell, and Maxwell fluids filling the porous space all can be deduced as the special cases. We also discuss the velocity and temperature fields of the generalized second grade fluid through a porous medium performing the same motion, from which the Newtonian solutions can be easily deduced.

The graphs of the velocity field indicate that the potential velocity decays in time and space and the strength of the potential vortex is directly proportional to parameters $\lambda$ and

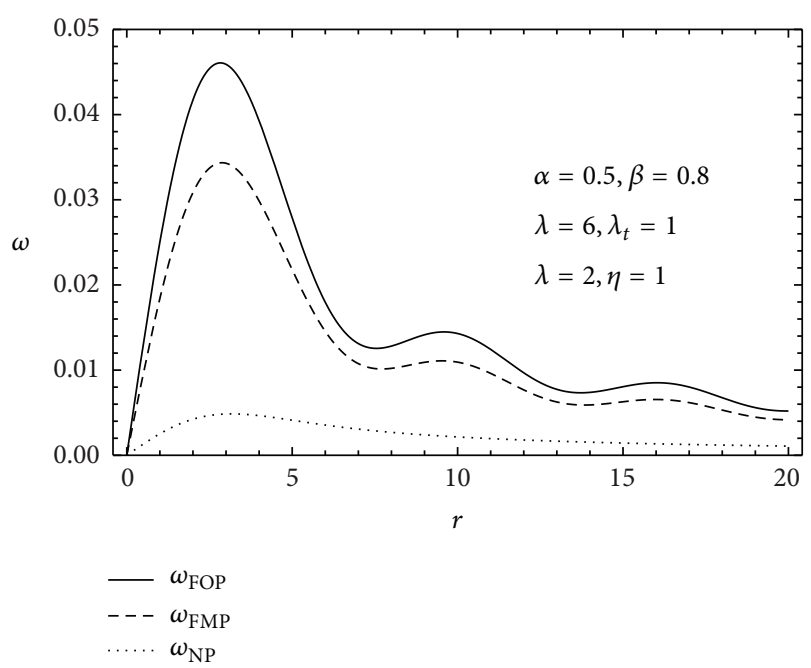

FIGURE 6: Profiles of velocity $\omega(r, t)$ versus $r$, in a porous medium, for fractional Oldroyd-B fluids (curve $\omega_{\mathrm{FOP}}$ ), fractional Maxwell fluids (curve $\omega_{\mathrm{FMP}}$ ), and Newtonian fluids (curve $\omega_{\mathrm{NP}}$ ).

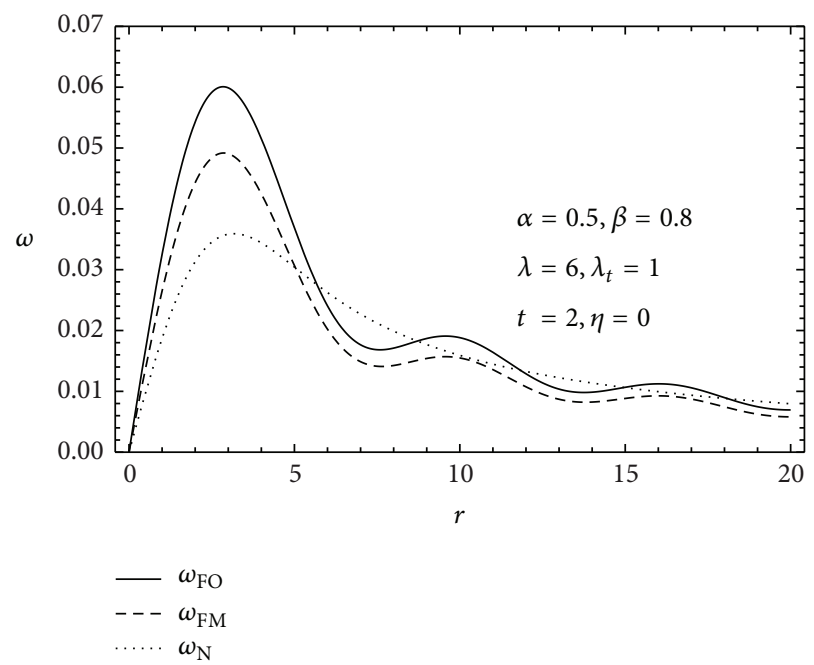

FIGURE 7: Profiles of velocity $\omega(r, t)$ versus $r$, in a nonporous medium, for fractional Oldroyd-B fluids (curve $\omega_{\mathrm{FO}}$ ), fractional Maxwell fluids (curve $\omega_{\mathrm{FM}}$ ), and Newtonian fluids (curve $\omega_{\mathrm{N}}$ ).

$\lambda_{t}$ and is inversely proportional to $\alpha$ and $\beta$. The vortexes of fractional Oldroyd-B fluid and fractional Maxwell fluid are much stronger than those of Newtonian one, no matter in porous or nonporous cases. The vortex in a nonporous medium is stronger than that in a porous medium.

\section{Conflict of Interests}

The author declares that there is no conflict of interests regarding the publication of this paper.

\section{Acknowledgments}

This work was supported by the National Natural Science Foundation of China (Grant no. 11201212), the Domestic 
Visiting Scholars Program funding for young core college teachers in Shandong province of China, and Applied Mathematics Enhancement Program of Linyi University.

\section{References}

[1] H. Frohlick and R. Sack, "Theory of rheological properties of dispersions," Proceedings of the Royal Society A, vol. 185, pp. 415430, 1946.

[2] J. G. Oldroyd, "On the formulation of rheological equations of state," Proceedings of the Royal Society A, vol. 200, pp. 523-541, 1950.

[3] K. R. Rajagopal and R. K. Bhatnagar, "Exact solutions for some simple flows of an Oldroyd-B fluid," Acta Mechanica, vol. 113, no. 1-4, pp. 233-239, 1995.

[4] T. Hayat, A. M. Siddiqui, and S. Asghar, "Some simple flows of an Oldroyd-B fluid," International Journal of Engineering Science, vol. 39, no. 2, pp. 135-147, 2001.

[5] C. Fetecau and C. Fetecau, "The first problem of Stokes for an Oldroyd-B fluid," International Journal of Non-Linear Mechanics, vol. 38, no. 10, pp. 1539-1544, 2003.

[6] C. Fetecau, "Analytical solutions for non-Newtonian fluid flows in pipe-like domains," International Journal of Non-Linear Mechanics, vol. 39, no. 2, pp. 225-231, 2004.

[7] M. Khan, T. Hayat, and S. Asghar, "Exact solution for MHD flow of a generalized Oldroyd-B fluid with modified Darcy's law," International Journal of Engineering Science, vol. 44, no. 5-6, pp. 333-339, 2006.

[8] M. Khan, K. Maqbool, and T. Hayat, "Influence of Hall current on the flows of a generalized Oldroyd-B fluid in a porous space," Acta Mechanica, vol. 184, no. 1-4, pp. 1-13, 2006.

[9] H. Qi and M. Xu, "Stokes' first problem for a viscoelastic fluid with the generalized Oldroyd-B model," Acta Mechanica Sinica, vol. 23, no. 5, pp. 463-469, 2007.

[10] D. Tong, X. Zhang, and X. Zhang, "Unsteady helical flows of a generalized Oldroyd-B fluid," Journal of Non-Newtonian Fluid Mechanics, vol. 156, no. 1-2, pp. 75-83, 2009.

[11] J. Zierep, Similarity Laws and Modeling, Marcel Dekker, New York, NY, USA, 1971.

[12] C. Fetecau and J. Zierep, "Decay of a potential vortex and propagation of a heat wave in a second grade fluid," International Journal of Non-Linear Mechanics, vol. 37, no. 6, pp. 1051-1056, 2002.

[13] F. Shen, W.-C. Tan, Y.-H. Zhao, and T. Masuoka, "Decay of vortex velocity and diffusion of temperature in a generalized second grade fluid," Applied Mathematics and Mechanics, vol. 25, no. 10, pp. 1053-11060, 2004.

[14] M. Khan, S. Hyder Ali, C. Fetecau, and H. Qi, "Decay of potential vortex for a viscoelastic fluid with fractional Maxwell model," Applied Mathematical Modelling, vol. 33, no. 5, pp. 2526-2533, 2009.

[15] C. Fetecau and C. Fetecau, "Decay of a potential vortex in an Oldroyd-B fluid," International Journal of Engineering Science, vol. 43, no. 3-4, pp. 340-351, 2005.

[16] C. Fetecau, C. Fetecau, M. Khan, and D. Vieru, "Decay of a potential vortex in a generalized Oldroyd-B fluid," Applied Mathematics and Computation, vol. 205, no. 1, pp. 497-506, 2008.

[17] W. Tan and T. Masuoka, "Stokes' first problem for an OldroydB fluid in a porous half space," Physics of Fluids, vol. 17, no. 2, Article ID 023101, 2005.
[18] M. El-Shahed and A. Salem, "Decay of vortex velocity and diffusion of temperature for fractional viscoelastic fluid through a porous medium," International Communications in Heat and Mass Transfer, vol. 33, no. 2, pp. 240-248, 2006.

[19] M. Husain, T. Hayat, and C. Fetecau, "A note on decay of potential vortex in an Oldroyd-B fluid through a porous space," Nonlinear Analysis: Real World Applications, vol. 10, no. 4, pp. 2133-2138, 2009.

[20] I. Podlubny, Fractional Differential Equations, vol. 198, Academic Press, San Diego, Calif, USA, 1999.

[21] I. N. Sneddon, Fourier Transforms, McGraw-Hill, Toronto, Canada, 1951. 


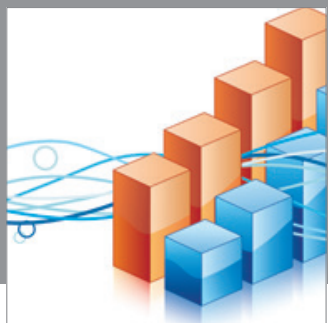

Advances in

Operations Research

mansans

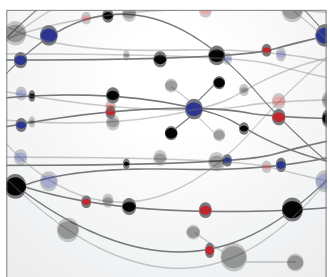

The Scientific World Journal
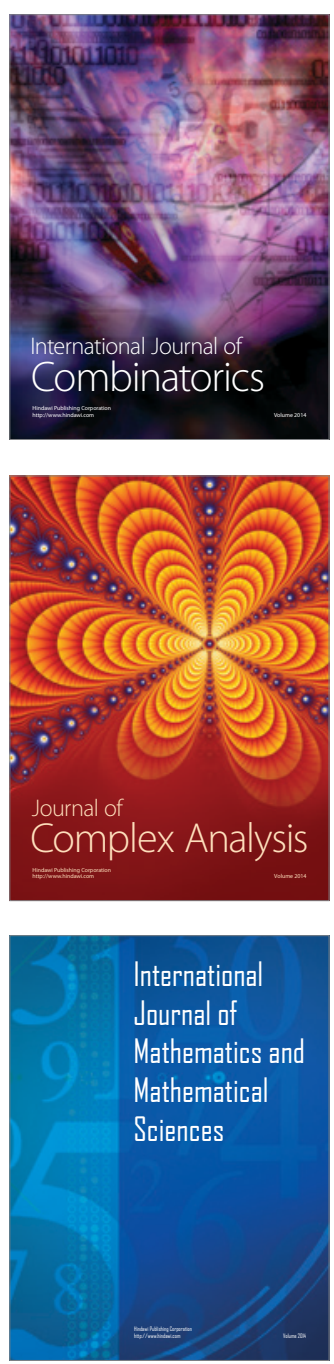
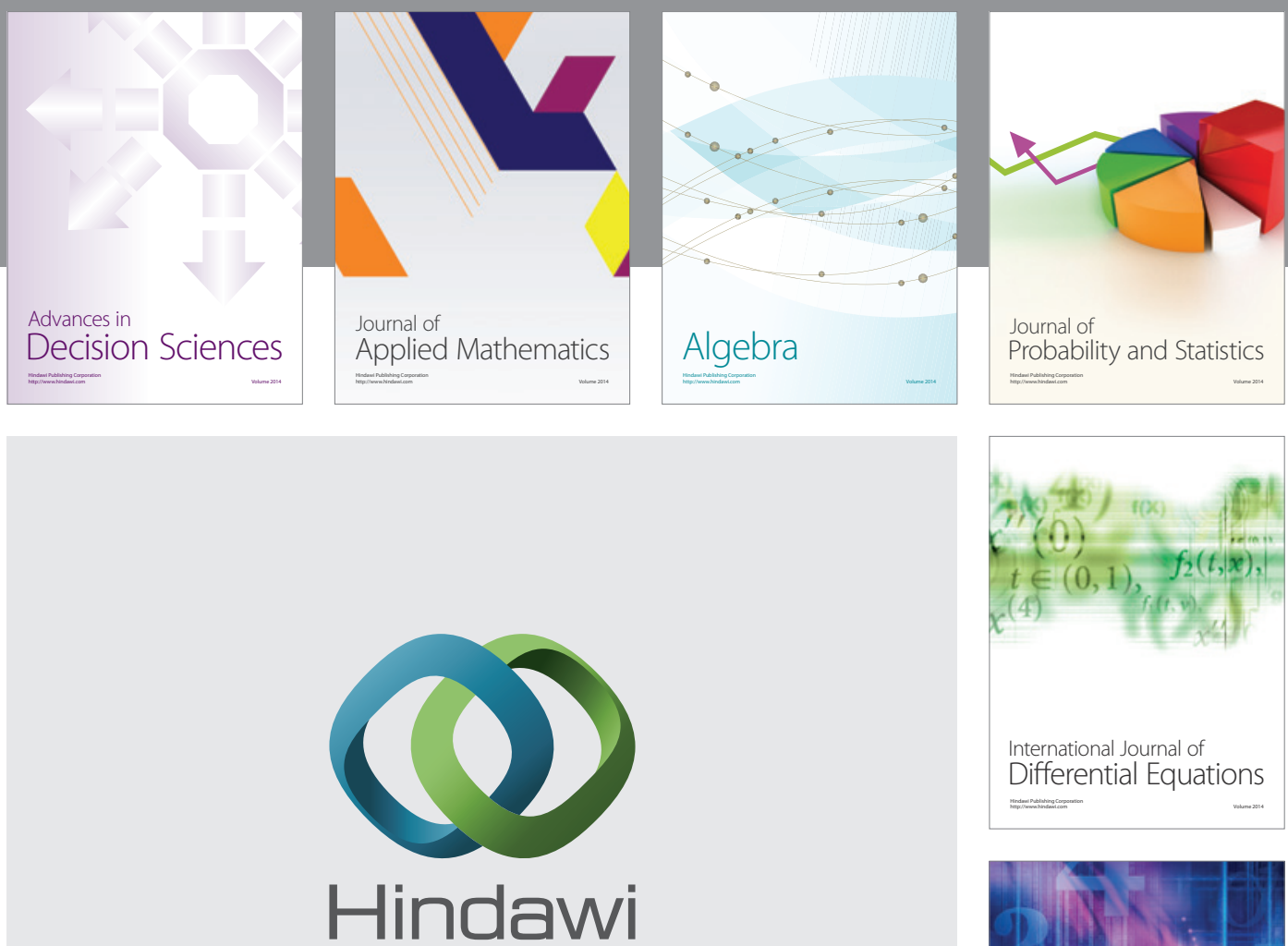

Submit your manuscripts at http://www.hindawi.com
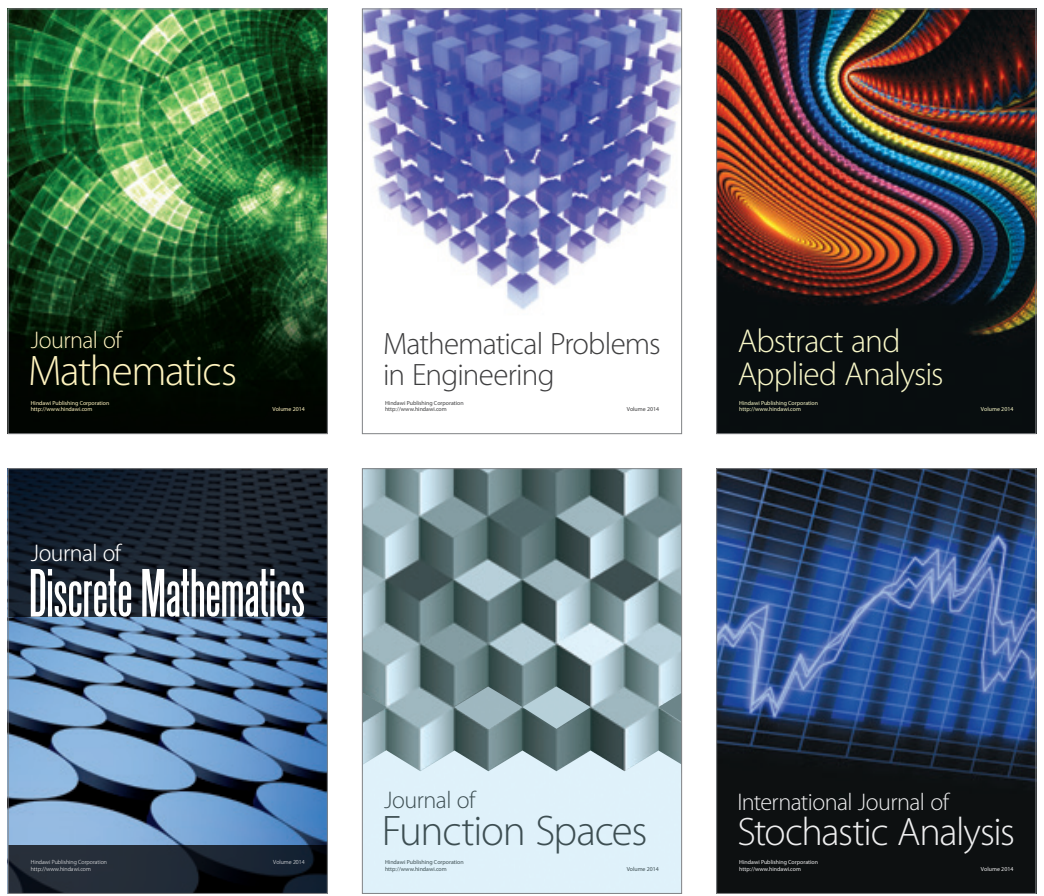

Journal of

Function Spaces

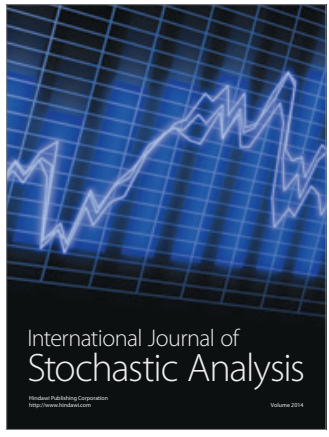

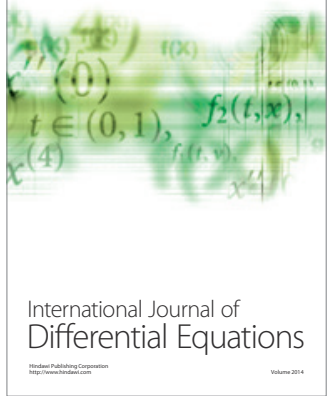
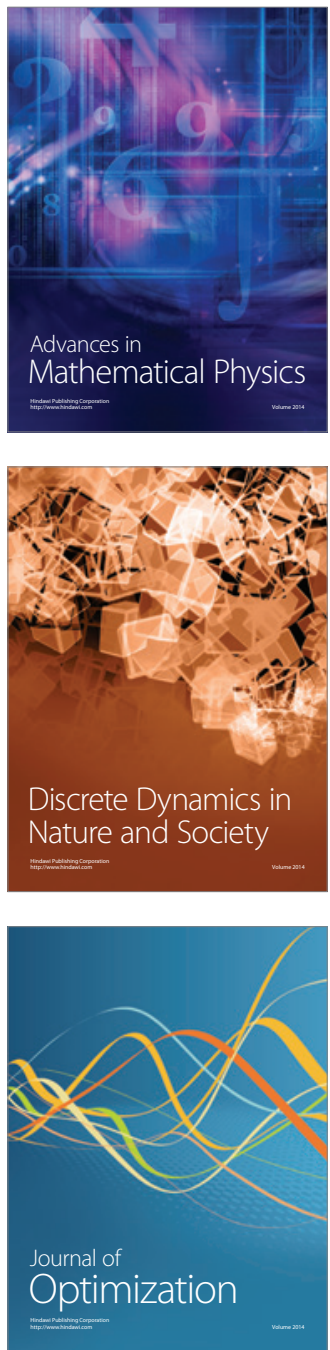